\title{
ACTIVATION OF THE GLYMPHATIC SYSTEM DURING SLEEP - IS THE CEREBRAL VENOUS OUTFLOW A MISSING PIECE OF THE PUZZLE?
}

\author{
Marian Simka \\ Department of Anatomy, Faculty of Medicine, University of Opole, Opole, Poland
}

EDITORIAL PAPER

Phlebological Review 2019; 27, 1: 1-2

D0l: https://doi.org/10.5114/pr.2019.93400

Submitted: 4.02 .2020

Accepted: 4.02 .2020

\author{
ADDRESS FOR CORRESPONDENCE \\ Marian Simka \\ Department of Anatomy \\ Faculty of Medicine \\ University of Opole \\ 11 A Kopernika PI. \\ 45-040 Opole, Poland \\ e-mail: mariansimka@poczta.onet.pl
}

Key words: glymphatic system, chronic cerebrospinal venous insufficiency, internal jugular vein.

Until recently, mechanisms responsible for activation of the astroglial-mediated interstitial fluid bulk flow, the so-called glymphatic system, were unclear, although it has been demonstrated that this system is primarily active during deep sleep [1-4]. Some researchers suggested that an increased pulsatility of the cerebral blood vessels could activate the glymphatic influx. However, the reason for this enhanced pulsatility, and why cerebral cleansing is more efficient during sleep, remained elusive. A recently published paper has finally shed some light on this problem [5]. It has been revealed that there is a temporary decrease of the cortical blood flow (measured by the BOLD fMRI) during the non-REM phase of sleep, which is followed by a macroscopic wave of inflow of the cerebrospinal fluid (CSF) at the level of the fourth ventricle. These haemodynamic and CSF flow oscillations are initiated by the slow-delta electrophysiological signalling.

Although during this study the function of the glymphatic system was not measured, it seems likely that aforementioned changes in the arterial and CSF flows are responsible for turning on the cerebral interstitial fluid bulk flow. Moreover, this important discovery suggests that an increased activity of the glymphatic system during deep sleep, which requires an enhanced flow of the CSF along the periarterial space surrounding the penetrating cerebral arteries, probably begins with the inflow of the
CSF into the cranial cavity from the vertebral canal, and that this inflow results from a decreased arterial volume inside the cranial cavity (the Kellie-Monroe doctrine). Such a mechanism would explain the need for sleep (at least regarding one of the functions of sleep). According to the results of the aforementioned study [5], an activation of the glymphatic system would require a temporary yet profound decrease in the activity of cortical neurons. Such a decrease would be difficult during the state of consciousness. However, in a deeply sleeping individual such coupled oscillations of the activity of neurons and the flow of the CSF would not interfere with physical activity or awareness.

In addition, the mechanisms of regulation of the activity of the glymphatic system, as suggested by Fultz et al. [5], would explain a possible role for impaired cerebral venous drainage in the pathophysiology of neurodegeneration. It has already been revealed that a pathology of veins draining the brain can be associated with a higher risk of neurodegenerative disorders. The glymphatic system is responsible for cleansing the cerebral parenchyma of waste products, and hence a higher risk of degeneration in the case of impaired glymphatic function $[3,6]$. Taking into account the Kellie-Monroe doctrine, proper regulation of the activity of the glymphatic system would require an undisturbed venous outflow. Otherwise, during the phase of diminished arterial flow there would 
be an increased pooling of venous blood inside the cranial cavity instead of the inflow of the CSF. Importantly, there are several reports linking an impaired cerebral venous outflow with neurodegenerative disorders [7-10], and papers demonstrating an association of an abnormal venous drainage with anomalous flow of the CSF [11-13]. Besides, it has already been suggested that abnormalities of the veins draining the brain, primarily lesions located extracranially, may be linked to neurodegeneration $[14,15]$. Now, with the report by Fultz et al., it seems easier to understand how such an abnormal venous outflow may result in neurodegenerative lesions of the brain. It might be speculated that in patients presenting with impaired cerebral venous drainage, because of such a compromised venous outflow, the oscillations of the cortical flow during the non-REM phase of sleep are not adequately accompanied by the changes of inflow of the CSF into the cranial cavity. This in turn would result in a less efficient cleansing of the brain parenchyma from pathological proteins, such as $\beta$-amyloid, $\alpha$-synuclein, or $\beta$-synuclein, and in a higher risk of neurodegenerative disorders.

As yet, venous flows into and out of the cranial cavity have not been studied in this context. Therefore, considering the existing evidence, investigations on cerebral venous haemodynamics should be one the directions of future research.

\section{The author declares no conflict of interest.}

\section{References}

1. Benveniste H, Heerdt PM, Fontes M, et al. Glymphatic system function in relation to anesthesia and sleep states. Anesth Analg 2019; 128: 747-758.

2. Lee H, Xie L, Yu M, et al. The effect of body posture on brain glymphatic transport. J Neurosci 2015; 35: 11034-11044.

3. Plog BA, Nedergaard M. The glymphatic system in the CNS health and disease: past, present and future. Annu Rev Pathol 2018; 13: 379-394.

4. Hablitz LM, Vinitsky HS, Sun Q, et al. Increased glymphatic influx is correlated with high EEG delta power and low heart rate in mice under anesthesia. Sci Adv 2019; 5: eaav5447.

5. Fultz NE, Bonmassar G, Setsompop K, et al. Coupled electrophysiological, hemodynamic, and cerebrospinal fluid oscillations in human sleep. Science 2019; 366: 628-631.

6. Liguori C, Chiaravalloti A, Izzi F, et al. Sleep apneas may represent a reversible risk factor for amyloid- $\beta$ pathology. Brain 2017; 140: e75.

7. Zamboni P, Galeotti R, Menegatti E, et al. Chronic cerebrospinal venous insufficiency in patients with multiple sclerosis. J Neurol Neurosurg Psychiatry 2009; 80: 392-399.

8. Zivadinov R, Marr K, Cutter G, et al. Prevalence, sensitivity, and specificity of chronic cerebrospinal venous insufficiency in MS. Neurology 2011; 77: 138-144.
9. Beggs C, Chung CP, Bergsland N, et al. Jugular venous reflux and brain parenchyma volumes in elderly patients with mild cognitive impairment and Alzheimer's disease. BMC Neurology 2013; 13: 157.

10. Chung CP, Beggs C, Wang PN, et al. Jugular venous reflux and white matter abnormalities in Alzheimer's disease: a pilot study. J Alzheimers Dis 2014; 39: 601-609.

11. Beggs CB, Magnano C, Shepherd SJ, et al. Aqueductal cerebrospinal fluid pulsatility in healthy individuals is affected by impaired cerebral venous drainage. J Magn Imaging 2014; 40: 1215-1222.

12. Beggs $\mathrm{CB}$, Magnano $\mathrm{C}$, Belov $\mathrm{P}$, et al. Internal jugular vein cross-sectional area and cerebrospinal fluid pulsatility in the aqueduct of Sylvius: a comparative study between healthy subjects and multiple sclerosis patients. PLoS One 2016; 11: e0153960.

13. Beggs CB, Shepherd SJ, Cecconi P, Lagana M. Predicting the aqueductal cerebrospinal fluid pulse: a statistical approach. Appl Sci 2019; 9: 2131.

14. Simka M, Skuła M. Potential involvement of impaired venous outflow from the brain in neurodegeneration: lessons learned from the research on chronic cerebrospinal venous insufficiency. Rev Recent Clin Trials 2019; 14: 235-236.

15. Zivadinov R, Chung CP. Potential involvement of the extracranial venous system in central nervous system disorders and aging. BMC Med 2013; 11: 260. 